\title{
Prenatal and postnatal detection of congenital heart diseases
}

\author{
Agata Szpera-Goździewicz ${ }^{1}$, Bartosz Burchardt², Martyna Musik³, \\ Grzegorz Henryk Bręborowicz' ${ }^{1}$, Mariola Ropacka-Lesiak1 \\ ${ }^{1}$ Department of Perinatology and Gynecology, Poznan University of Medical Sciences, Poznan, Poland \\ 2Department of Surgical Gynecology, Poznan University of Medical Sciences, Poznan, Poland \\ ${ }^{3}$ Department of Dermatology, Venerology and Allergology, University Clinical Centre in Gdansk, Poland
}

\section{INTRODUCTION}

Congenital heart defects are among the most common birth defects and represent a major challenge in prenatal diagnosis and therapy of a newborn.

\section{CASES PRESENTATION}

The results of prenatal foetal heart examinations, which were performed at the Ultrasonographic Laboratory in the years 2013 to 2014, were subjected to retrospective analysis. Incompatibility of diagnoses was evaluated based on the modified classification created by Berkley et al., who distinguished the so-called "large" and "small" differences. "Large" differences resulted in the necessity of a sudden change in the child care program, implementation of urgent highly-specialized interventions available in the centres with the highest degree of referentiality and thus determining the place of delivery. On the other hand, "small" differences did not imply the need for such changes in the newborn care program.

Forty-nine foetal heart defects were analyzed. The abnormal four chamber view in routinely performed ultrasonography was the most common indication (71.4\%) for prenatal echocardiography. The mean gestational age at the time of the first consultation was 30 weeks. The most frequently observed defects include: ventricular septal defect (20 cases), atrioventricular septal defect (7 cases), coarctation of the aorta (5 cases) and hypoplastic left heart syndrome (4 cases). There was no intrauterine death reported and verification of the type of defect took place within two days after birth.

Prenatal diagnosis was consistent with postnatal diagnosis in $76.2 \%$. In $11.1 \%$ defects detected in utero differed from post-natal diagnoses (Table 1). All differences in pre- and post-natal diagnosis were classified as "small". Furthermore,

Table 1. Non-compliance of pre- and postnatal diagnosis and classification into "small" and "large" differences

\begin{tabular}{|l|l|l|l|}
\hline Prenatal diagnosis & Postnatal diagnosis & Management & $\begin{array}{l}\text { Classification of diagnoses } \\
\text { non-compliance }\end{array}$ \\
\hline ToF & Pulmonary valve stenosis & Surgery vs. valvuloplasty of pulmonary valve & Small \\
\hline VSD & AVSD & Expectant vs. elective surgery & Small \\
\hline AVSD & VSD & Elective surgery vs. expectant & Small \\
\hline TGA + VSD & DORV & Surgical treatment in both cases & Small \\
\hline DORV & Dextroposition of the aorta + VSD & Surgery vs. expectant & Small \\
\hline AVSD & VSD & Elective surgery vs. expectant & Small \\
\hline $\begin{array}{l}\text { VSD } \\
\text { ToF - tetralogy of Fallot; VSD - ventricular septal defect; AVSD } \\
\text { outlet right ventricle }\end{array}$ & AVSD & -atrioventricular septal defect; TGA - transposition of the great arteries; DORV - double & Small \\
\hline
\end{tabular}

Corresponding author:

Agata Szpera-Goździewicz

Department of Perinatology and Gynecology

Poznan University of Medical Sciences

33 Polna St., 60-535 Poznan, Poland

e-mail: agata.szpera@gmail.com 
in 8 cases (12.7\%) false positive diagnoses of heart defects were made. Most often they related to the defect in the interventricular septum and coarctation of the aorta ( 2 and 3 cases, respectively).

Many studies analyze the compliance of prenatal and postnatal diagnoses, but only some of them focus on a thorough analysis of differences. Berkley et al. analyzed 53 echocardiograms performed both pre- and postnatally, which showed 3 cases (5.7\%) of "small" differences and 3 cases (5.7\%) of "large" differences. In our study, we observed only "small" differences. Discrepancies classified as "small" do not considerably change the management after birth. Discrepancies classified as "large" prevents in utero transport to centres providing proper care in the postnatal period, which in turn may lead to the delay of aid and worsening of prognosis.

The studies conducted by Seulgi et al. demonstrated complete compliance between pre- and post-natal diagnosis in $88.5 \%$. Meyer-Wittkopf et al. showed complete compliance of such diagnoses in complex heart defects in $59 \%$ of cases, if the test was performed by obstetricians and in $95 \%$ of cases if such an examination was conducted by paediatric cardiologists. In our material, this percentage is similar to that described in the literature.

\section{CONCLUSIONS}

Prenatal foetal heart evaluation performed by a well-trained obstetricians is a very useful tool for the identification of fetal congenital heart defects. 